\title{
Reversed L-shaped Association Between Baseline Brachial-ankle Pulse Wave Velocity and Short-Term Risk of First Stroke in Patients With Hypertension: A Population-based Cohort Study
}

\section{Lihua Hu}

Peking University First Hospital

Chonglei Bi

People's Hospital of Rongcheng, Shangdon

\section{Lishun Liu}

China Agricultural University

\section{Yun Song}

China Agricultural University

\section{Yue Zhang}

China Pharmaceutical University

\section{Binyan Wang}

Anhui Medical University

\section{Chongqian Fang}

People's Hospital of Rongcheng

\section{Hai Ma}

Family Planning Association of Uganda: Reproductive Health Uganda

\section{Xiao Huang}

Nanchang University Second Affiliated Hospital

\section{Yan Zhang}

Peking University First Hospital

Huihui Bao

Nanchang University Second Affiliated Hospital

\section{Ping Li}

Nanchang University Second Affiliated Hospital

\section{Xiping Xu}

China Agricultural University

\section{Xiaobin Wang}

Johns Hopkins University - Homewood Campus: Johns Hopkins University

\section{Xiaoshu Cheng}




\section{Yong Huo}

Peking University First Hospital

Jianping Li ( $\square$ lijianping03455@pkufh.com )

Peking University First Hospital

\section{Original investigation}

Keywords: brachial-ankle pulse wave velocity, stroke, reversed L-shaped curve, hypertension, short-term risk

Posted Date: October 29th, 2020

DOI: https://doi.org/10.21203/rs.3.rs-97466/v1

License: (c) (i) This work is licensed under a Creative Commons Attribution 4.0 International License.

Read Full License 


\section{Abstract}

Background: Brachial-ankle pulse wave velocity (baPWV) is available as a noninvasive measure of arterial stiffness. However, little information is available on the association between baPWV and the short-term risk of stroke in patients with hypertension. Therefore, our current study aimed to assess the association between baseline baPWV and short-term risk of first stroke. We were particularly interested in identifying those individuals at high risk of developing stroke among patients with hypertension.

Methods: From January 1, 2017, to December 31, 2017, a total of 9787 individuals without preexisting stroke from the China Hypertension Registry Study were included in this analysis. Follow-up continued until December 31, 2018. The primary outcome was first stroke. The crude and adjusted risks of first stroke were estimated by hazard ratios (HRs) and 95\% Cls using Cox proportional hazards models, without or with adjusting for pertinent covariates, respectively.

Results: During a median follow-up of 20.8 months, there were 138 total first strokes including 123 first ischemic strokes and 15 first hemorrhagic strokes. Smooth curve showed that the relationship between baPWV and risk of first stroke and first ischemic stroke was curvilinear and reversed L-shaped. High baPWV levels ( $\geq 21.31 \mathrm{~m} / \mathrm{s}$ ) was associated with increased risk of first stroke (HR $=1.52 ; 95 \% \mathrm{Cl}$ : 1.05 2.21) and first ischemic stroke ( $\mathrm{HR}=1.53 ; 95 \% \mathrm{Cl}$ : 1.03-2.26) compared to low baPWV levels $(<21.31$ $\mathrm{m} / \mathrm{s}$ ). E-value analysis suggested robustness to unmeasured confounding. Our findings may propose the applicability of baPWV measurement for prediction of stroke development, especially in subjects with a low cardiovascular (CVD) risk.

Conclusions: High baPWV levels ( $\geq 21.31 \mathrm{~m} / \mathrm{s}$ ) were associated with increased the short-term risk of first stroke among Chinese hypertensive adults, compared to low baPWV levels, supporting a reversed Lshaped association. Our findings warrant additional investigation.

\section{Introduction}

Stroke is the leading cause of death in China and the second leading cause of death in the world ${ }^{[1,2]}$. Primary prevention is regarded as the best option to reduce the population burden of stroke ${ }^{[3]}$.

Specifically, hypertension is a well-recognized major modifiable risk factor for stroke $\mathrm{e}^{[4]}$. Despite great efforts to identify traditional risk factors and interventions focusing on modifying unhealthy lifestyles, rising stroke incidence and mortality are still observed in China. Therefore, there is an urgent need to identify novel modifiable risk factors to inform the primary prevention of stroke and to identify those individuals at highest risk of developing stroke among patients with hypertension.

Arteriosclerosis is a recognized predictor of cardiovascular diseases and stroke $\mathrm{e}^{[5-7]}$. Carotid-femoral pulse wave velocity (cfPWV) is the gold standard for measuring large arterial stiffness. As it is time consuming to measure, involves manipulation of the femoral artery area, and has bad reproducibility with requiring a highly skilled technician, the brachial-ankle pulse wave velocity (baPWV) is launched. BaPWV 
is available as a noninvasive measure of central and peripheral arterial stiffness ${ }^{[8]}$. Moreover, baPWV has been reported to be closely correlated with the directly measured cfPWV ${ }^{[9]}$. The measurement of baPWV has been proposed for the early detection of vascular damage in hypertensive patients ${ }^{[10]}$.

Indeed growing evidence has established the associations of baPWV with uncontrolled blood pressure (BP), cardiovascular mortality and morbidity among the general population ${ }^{[11-14]}$. Although few studies have found a relationship between increased baPWV and incidence of stroke $e^{[12,15,16]}$, the reference values for baPWV are still unknown in healthy populations and patients at increased cardiovascular risk. Additionally, whether baPWV maintains a predictive value in hypertensive patients requires further investigation, for evidence on the association is limited.

Therefore, our current study aimed to assess the association between baseline baPWV and short-term risk of first stroke using data from China Hypertension Registry Study (CHRS). We were particularly interested in identifying those individuals at high risk of developing stroke among patients with hypertension.

\section{Methods}

\section{Study design and population}

Our present study is a subset of the CHRS which was an ongoing community-based non-intervention, prospective, observational, multicenter, real-world registry study and was mainly conducted in Rongcheng county, Shandong province, Lianyungang, Jiangsu province, China and Wuyuan county, Jiangxi province. The CHRS study recruited consecutive consenting individuals with hypertension (untreated or treated with antihypertensive drugs) or with suspected hypertension. It was designed to assess the related factors of hypertension affecting its prognosis, and finally to construct the risk prediction model of cardio-cerebral and renal vascular diseases. Exclusion criteria were: psychological or nervous system impairment and inability to provide informed consent or unwillingness/inability to adhere to study protocol. All participants were asked to perform the epidemiological questionnaire and physical examination. The study was approved by the Ethics Committee of the Institute of Biomedicine, Anhui Medical University, Hefei, China. All participants signed an approved written consent after it was explained to them.

The current study utilized data from the Rongcheng CHRS. From January 2017 to December 2017, 111297 patients with hypertension or with suspected hypertension in Shandong Rongcheng were screened, and we collected their baseline general information. 10935 participants were randomly selected for baPWV assessment. Next, we selected participants according to the exclusion criteria listed below: ankle brachial index $(A B I)<0.90(n=638)$, having history of stroke $(n=385)$, and having history of atrial fibrillation $(n=125)$.

\section{BaPWV measurement}


In this study, the exposure variable was baseline baPWV. BaPWV was automatically measured byPWV/ABI instruments (form PWV/ABI, BP-203RPE; Omron-Colin, Japan) ${ }^{[17]}$. The validation of this automatic device and its reproducibility have been previously published ${ }^{[18]}$. Occlusion and monitoring cuffs matched with oscillometric sensors were wrapped around subjects' arms and the ankles, and pulse volume wave forms of the bilateral brachial and posterior tibial arteries were recorded simultaneously to determine the time interval between the initial increase in brachial and tibial waveforms (the transit time, Tba). The transmission distance from the brachium to ankle was calculated according to body height. The path length from the suprasternal notch to the brachium $(\mathrm{Lb})$ was obtained using the following equation: $L b=0.2159 \times$ height of the patient $(\mathrm{cm})-2.0734$. The path length from the suprasternal notch to the ankle ( $\mathrm{La})$ was obtained using the following equation: $\mathrm{La}=0.8129 \times$ height of the patient $(\mathrm{cm})+$ 12.328. And the baPWV value was calculated as the ratio of transmission distance from the brachium to ankle divided by the transit time: baPWV $=(\mathrm{La}-\mathrm{Lb}) / \mathrm{Tba}$. The maximum of the right and left-side baPWV values was used for final analysis ${ }^{[18]}$.

\section{Outcomes}

The primary outcome of this study was a first nonfatal or fatal stroke. Secondary outcomes included first ischemic stroke (fatal and nonfatal) and first hemorrhagic stroke (fatal and nonfatal). Data were linked to stroke data through December 31st, 2018. The short-term stroke Short-term stroke occurred within 2 years of follow-up. Information on incidence of stroke for all participants was obtained via the Center for Disease Control and Prevention of Rongcheng and checked against the national health insurance system with electronic linkage to all hospitalizations, or ascertained through active follow-up. Diseases were coded according to the International Classification of Diseases, Tenth Revision. Secondary outcomes included first ischemic stroke (163) and first hemorrhagic stroke (160-161). The primary outcome (first nonfatal or fatal stroke) included first ischemic stroke (163), first hemorrhagic stroke (160-161), and no type stroke (164).

Local authorities from medical institutions are required to report all new cases of stroke to the local Center for Disease Control and Prevention. A report card which includes information on demographics, diagnostic basis, and date of stroke is required to be submitted on the 28th of each month. Quality control, including finding and deleting repeated cases, error checking, and determining any missed cases, is completed by trained officials. Furthermore, the local Center for Disease Control and Prevention is also responsible for deleting repeated cases and finding logistical errors and missed cases. In addition, $5 \%$ of all uploaded cases are randomly chosen for further confirmation by phone or door-to-door interviews.

\section{Covariates}

At this baseline assessment, participants reported on their lifestyle, medical conditions and demographic information; medical history, health status, and medication intake were queried by trained health professionals. The anthropometric examinations included weight, height, waist circumference and hip circumference. Body mass index (BMI) was calculated as the body weight in kilograms divided by the square of the height in meters $\left(\mathrm{kg} / \mathrm{m}^{2}\right)$. Variables thought to be confounders based on existing literature 
and clinical judgment were included. Therefore, the following variables were used to construct the fully adjusted model. Continuous variables included age (years), BMI $\left(\mathrm{kg} / \mathrm{m}^{2}\right)$, systolic blood pressure (SBP, $\mathrm{mmHg}$ ) and diastolic blood pressure (DBP, $\mathrm{mmHg}$ ). Categorical variables consisted of sex, smoking (current smoker, former smoker or never smoker), drinking (current drinker, former drinker or never drinker), education status (less than high school, high school/equivalent, or greater than high school), labor intensity (light, moderate or heavy), sleep quality (poor, moderate or good), self-reported history of diseases [hypertension, diabetes, hyperlipidemia and coronary heart disease (CHD)], medication use (antihypertensive drugs, glucose-lowering drugs, lipoprotein-lowering drugs and antiplatelet drugs). Hypertension was defined by $\geq 1$ of the following criteria: systolic blood pressure $\geq 140 \mathrm{~mm} \mathrm{Hg}$ or diastolic blood pressure $\geq 90$ or self-reported history of hypertension or use of medications to treat hypertension ${ }^{[20]}$.

\section{Statistical analysis and Sensitivity analysis}

Data are presented as mean \pm SD for continuous variables and as frequency (\%) for categorical variables. The population characteristics by baPWV quartiles were compared using analysis of Variance (ANOVA) tests (continuous variables), or $\chi^{2}$ tests (categorical variables), accordingly. Crude cumulative incidence across baPWV quartiles was estimated using Kaplan-Meier curves. Restricted cubic spline, and Cox proportional hazards models were performed to evaluate the association between baseline baPWV and first stroke and its subtypes. Potential confounders included in the Cox proportional hazards models were all adjusted for the same covariates: age, sex, BMI, education status, smoking status, drinking status, labor intensity, sleep quality, hypertension, self-reported of diabetes, self-reported hyperlipidemia, selfreported CHD, antihypertensive drugs, glucose-lowering drugs, lipoprotein-lowering drugs and antiplatelet drugs. BaPWV was evaluated using models for categorical variables according to quartiles. The lowest quartile (Q1) of baPWV was considered as a reference group. The crude and adjusted hazard ratios (HRs) and their $95 \%$ confidence intervals (Cls) were estimated by the Cox proportional hazards regression model.

The robustness of these findings was assessed in multiple sensitivity analyses. First, we explored the potential for unmeasured confounding between baPWV and first stroke by calculating E-values ${ }^{[21]}$. The Evalue quantifies the required magnitude of an unmeasured confounder that couldnegate the observed association between baPWV and first stroke. Second, in further exploratory analyses, potential effect modifications of the association between baPWV and first stroke were assessed for the variables: age (< 65 vs. 65 years), sex, BMl (<25 vs. $25 \mathrm{~kg} / \mathrm{m}^{2}$ ), SBP (<140 vs. $140 \mathrm{~mm} \mathrm{Hg}$ ), DBP (<90 vs. $90 \mathrm{~mm} \mathrm{Hg}$ ), smoking status, sleep quality, self-reported diabetes, self-reported hyperlipidemia and self-reported CHD.

All analyses were performed using the statistical package R (http://www.R-project.org, The R Foundation) and a 2-sided type I error probability of 0.05 .

\section{Results}




\section{Baseline characteristics of study participants}

As shown in Fig. 1, a total of 9787 participants were included in this analysis. The baseline characteristics for all participants and by baPWV quartiles are listed in Table 1. The mean age was $66.7 \pm$ 7.9 years, and $34.4 \%$ of subjects were men $(n=3363)$. $97.3 \%(9521 / 9787)$ were hypertensive patients. The mean baPWV level at baseline was $19.1 \pm 4.5 \mathrm{~m} / \mathrm{s}$. A higher baPWV value was associated with female sex, older age, higher blood pressures, higher presence of hypertension, diabetes mellitus, CHD and higher use of glucose-lowering drugs. BMl, current smoking, education level, labor intensity, history of hyperlipidemia and use of lipoprotein-lowering drugs were negatively associated with baPWV. 
Table 1

Baseline characteristics of participants according to baseline baPWV quartiles

\begin{tabular}{|c|c|c|c|c|c|c|}
\hline \multirow[t]{3}{*}{ Characteristics ${ }^{*}$} & \multirow[t]{3}{*}{ Total } & \multicolumn{5}{|c|}{ BaPWV, m/s } \\
\hline & & Q1 & Q2 & Q3 & Q4 & $P$ \\
\hline & & $(<16.00)$ & $\begin{array}{l}(16.00- \\
18.33)\end{array}$ & $\begin{array}{l}(18.33- \\
21.31)\end{array}$ & $(\geq$ & \\
\hline$N$ & 9787 & 2436 & 2455 & 2445 & 2451 & \\
\hline Sex, n (\%) & & & & & & $\begin{array}{l}< \\
0.001\end{array}$ \\
\hline Male & $\begin{array}{l}3363 \\
(34.4)\end{array}$ & $\begin{array}{l}918 \\
(37.7)\end{array}$ & $\begin{array}{l}857 \\
(34.9)\end{array}$ & 816 (33.4) & $\begin{array}{l}772 \\
(31.5)\end{array}$ & \\
\hline Female & $\begin{array}{l}6424 \\
(65.6)\end{array}$ & $\begin{array}{l}1518 \\
(62.3)\end{array}$ & $\begin{array}{l}1598 \\
(65.1)\end{array}$ & $\begin{array}{l}1629 \\
(66.6)\end{array}$ & $\begin{array}{l}1679 \\
(68.5)\end{array}$ & \\
\hline Age, y & $\begin{array}{l}66.7 \pm \\
7.9\end{array}$ & $\begin{array}{l}62.3 \pm \\
7.7\end{array}$ & $65.3 \pm 7.3$ & $67.9 \pm 6.8$ & $\begin{array}{l}71.3 \pm \\
7.0\end{array}$ & $<.001$ \\
\hline $\mathrm{BMI}, \mathrm{kg} / \mathrm{m}^{29}$ & $\begin{array}{l}27.1 \pm \\
3.7\end{array}$ & $\begin{array}{l}27.6 \pm \\
3.8\end{array}$ & $27.3 \pm 3.7$ & $27.0 \pm 3.6$ & $\begin{array}{l}26.5 \pm \\
3.6\end{array}$ & $\begin{array}{l}<.001 \\
0.001\end{array}$ \\
\hline SBP, mm Hg & $\begin{array}{l}155.8 \pm \\
19.5\end{array}$ & $\begin{array}{l}148.4 \pm \\
17.7\end{array}$ & $\begin{array}{l}153.6 \pm \\
17.9\end{array}$ & $\begin{array}{l}157.9 \pm \\
18.7\end{array}$ & $\begin{array}{l}163.3 \pm \\
20.6\end{array}$ & $\begin{array}{l}< \\
0.001\end{array}$ \\
\hline DBP, mm Hg & $\begin{array}{l}88.0 \pm \\
10.8\end{array}$ & $\begin{array}{l}87.7 \pm \\
10.4\end{array}$ & $\begin{array}{l}87.8 \pm \\
10.7\end{array}$ & $87.7 \pm 10.7$ & $\begin{array}{l}88.8 \pm \\
11.4\end{array}$ & $\begin{array}{l}<.001 \\
0.001\end{array}$ \\
\hline $\mathrm{BaPWV}, \mathrm{m} / \mathrm{s}$ & $\begin{array}{l}19.1 \pm \\
4.5\end{array}$ & $\begin{array}{l}14.5 \pm \\
1.2\end{array}$ & $17.2 \pm 0.7$ & $19.7 \pm 0.8$ & $\begin{array}{l}25.2 \pm \\
4.1\end{array}$ & $\begin{array}{l}< \\
0.001\end{array}$ \\
\hline Smoking status, n (\%) & & & & & & $\begin{array}{l}<.001 \\
0.001\end{array}$ \\
\hline Never & $\begin{array}{l}7815 \\
(79.9)\end{array}$ & $\begin{array}{l}1898 \\
(77.9)\end{array}$ & $\begin{array}{l}1946 \\
(79.3)\end{array}$ & $\begin{array}{l}1965 \\
(80.4)\end{array}$ & $\begin{array}{l}2006 \\
(81.8)\end{array}$ & \\
\hline
\end{tabular}

Q1: quartile 1 (lowest quartile); Q2: quartile 2; Q3: quartile 3; Q4: quartile 4 (highest quartile).

Abbreviations: BaPWV, brachial-ankle pulse wave velocity; BMI, body mass index; SBP, systolic blood pressure; DBP, diastolic blood pressure; CHD, coronary heart disease.

${ }^{*}$ Data are presented as number (\%) or mean \pm SD.

'BMI was calculated as the body weight in kilograms divided by the square of the height in meters $\left(\mathrm{kg} / \mathrm{m}^{2}\right)$.

${ }^{\dagger}$ Hypertension was defined by $\geq 1$ of the following criteria: systolic blood pressure $\geq 140 \mathrm{~mm} \mathrm{Hg}$ or diastolic blood pressure $\geq 90$ or self-reported history of hypertension or use of medications to treat hypertension. 


\begin{tabular}{|c|c|c|c|c|c|c|}
\hline \multirow[t]{3}{*}{ Characteristics* } & \multirow[t]{3}{*}{ Total } & \multicolumn{5}{|c|}{ BaPWV, m/s } \\
\hline & & Q1 & Q2 & Q3 & Q4 & $P$ \\
\hline & & $(<16.00)$ & $\begin{array}{l}(16.00- \\
18.33)\end{array}$ & $\begin{array}{l}(18.33- \\
21.31)\end{array}$ & $\left(\geq \frac{\geq}{21.31)}\right.$ & \\
\hline Former & $687(7.0)$ & $162(6.7)$ & $189(7.7)$ & $161(6.6)$ & $175(7.1)$ & \\
\hline Current & $\begin{array}{l}1284 \\
(13.1)\end{array}$ & $\begin{array}{l}376 \\
(15.4)\end{array}$ & $\begin{array}{l}320 \\
(13.0)\end{array}$ & $318(13.0)$ & $\begin{array}{l}270 \\
(11.0)\end{array}$ & \\
\hline Drinking status, n (\%) & & & & & & 0.685 \\
\hline Never & $\begin{array}{l}7863 \\
(80.3)\end{array}$ & $\begin{array}{l}1935 \\
(79.4)\end{array}$ & $\begin{array}{l}1991 \\
(81.1)\end{array}$ & $\begin{array}{l}1964 \\
(80.3)\end{array}$ & $\begin{array}{l}1973 \\
(80.5)\end{array}$ & \\
\hline Former & $260(2.7)$ & $73(3.0)$ & $65(2.6)$ & $57(2.3)$ & $65(2.7)$ & \\
\hline Current & $\begin{array}{l}1664 \\
(17.0)\end{array}$ & $\begin{array}{l}428 \\
(17.6)\end{array}$ & $\begin{array}{l}399 \\
(16.3)\end{array}$ & $424(17.3)$ & $\begin{array}{l}413 \\
(16.9)\end{array}$ & \\
\hline Education, n (\%) & & & & & & $\begin{array}{l}<.001 \\
0.001\end{array}$ \\
\hline$<$ High school & $\begin{array}{l}8776 \\
(89.7)\end{array}$ & $\begin{array}{l}2072 \\
(85.1)\end{array}$ & $\begin{array}{l}2162 \\
(88.1)\end{array}$ & $\begin{array}{l}2236 \\
(91.5)\end{array}$ & $\begin{array}{l}2306 \\
(94.1)\end{array}$ & \\
\hline High school & $927(9.5)$ & $\begin{array}{l}336 \\
(13.8)\end{array}$ & $\begin{array}{l}269 \\
(11.0)\end{array}$ & $185(7.6)$ & $137(5.6)$ & \\
\hline$>$ High school & $84(0.9)$ & $28(1.1)$ & $24(1.0)$ & $24(1.0)$ & $8(0.3)$ & \\
\hline Labor intensity, n (\%) & & & & & & $\begin{array}{l}< \\
0.001\end{array}$ \\
\hline Light & $\begin{array}{l}6551 \\
(66.9)\end{array}$ & $\begin{array}{l}1553 \\
(63.8)\end{array}$ & $\begin{array}{l}1630 \\
(66.4)\end{array}$ & $\begin{array}{l}1648 \\
(67.4)\end{array}$ & $\begin{array}{l}1720 \\
(70.2)\end{array}$ & \\
\hline Moderate & $\begin{array}{l}2426 \\
(24.8)\end{array}$ & $\begin{array}{l}651 \\
(26.7)\end{array}$ & $\begin{array}{l}629 \\
(25.6)\end{array}$ & $586(24.0)$ & $\begin{array}{l}560 \\
(22.8)\end{array}$ & \\
\hline
\end{tabular}

Q1: quartile 1 (lowest quartile); Q2: quartile 2; Q3: quartile 3; Q4: quartile 4 (highest quartile).

Abbreviations: BaPWV, brachial-ankle pulse wave velocity; BMI, body mass index; SBP, systolic blood pressure; DBP, diastolic blood pressure; CHD, coronary heart disease.

*Data are presented as number (\%) or mean \pm SD.

'BMI was calculated as the body weight in kilograms divided by the square of the height in meters $\left(\mathrm{kg} / \mathrm{m}^{2}\right)$.

${ }^{\dagger}$ Hypertension was defined by $\geq 1$ of the following criteria: systolic blood pressure $\geq 140 \mathrm{~mm} \mathrm{Hg}$ or diastolic blood pressure $\geq 90$ or self-reported history of hypertension or use of medications to treat hypertension. 


\begin{tabular}{|c|c|c|c|c|c|c|}
\hline \multirow[t]{3}{*}{ Characteristics* } & \multirow[t]{3}{*}{ Total } & \multicolumn{5}{|c|}{ BaPWV, m/s } \\
\hline & & \multirow{2}{*}{$\begin{array}{l}\text { Q1 } \\
(<16.00)\end{array}$} & \multirow{2}{*}{$\begin{array}{l}\text { Q2 } \\
(16.00- \\
18.33)\end{array}$} & \multirow{2}{*}{$\begin{array}{l}\text { Q3 } \\
(18.33- \\
21.31)\end{array}$} & \multirow{2}{*}{$\begin{array}{l}\text { Q4 } \\
(\geq \\
21.31)\end{array}$} & \multirow{2}{*}{$\begin{array}{l}P \\
\text { value }\end{array}$} \\
\hline & & & & & & \\
\hline Heavy & $810(8.3)$ & $232(9.5)$ & $196(8.0)$ & $211(8.6)$ & $171(7.0)$ & \\
\hline Sleep quality, n (\%) & & & & & & 0.519 \\
\hline Poor & $\begin{array}{l}4973 \\
(50.8)\end{array}$ & $\begin{array}{l}1253 \\
(51.4)\end{array}$ & $\begin{array}{l}1241 \\
(50.5)\end{array}$ & $\begin{array}{l}1213 \\
(49.6)\end{array}$ & $\begin{array}{l}1266 \\
(51.7)\end{array}$ & \\
\hline Moderate & $\begin{array}{l}3066 \\
(31.3)\end{array}$ & $\begin{array}{l}761 \\
(31.2)\end{array}$ & $\begin{array}{l}762 \\
(31.0)\end{array}$ & $803(32.8)$ & $\begin{array}{l}740 \\
(30.2)\end{array}$ & \\
\hline Good & $\begin{array}{l}1748 \\
(17.9)\end{array}$ & $\begin{array}{l}422 \\
(17.3)\end{array}$ & $\begin{array}{l}452 \\
(18.4)\end{array}$ & $429(17.5)$ & $\begin{array}{l}445 \\
(18.2)\end{array}$ & \\
\hline Hypertension, $\mathrm{n}(\%)^{\dagger}$ & $\begin{array}{l}9521 \\
(97.3)\end{array}$ & $\begin{array}{l}2310 \\
(94.8)\end{array}$ & $\begin{array}{l}2394 \\
(97.5)\end{array}$ & $\begin{array}{l}2396 \\
(98.0)\end{array}$ & $\begin{array}{l}2421 \\
(98.8)\end{array}$ & $\begin{array}{l}<.001 \\
0.00\end{array}$ \\
\hline $\begin{array}{l}\text { Self-reported diabetes, } \mathrm{n} \\
(\%)\end{array}$ & $\begin{array}{l}1792 \\
(18.3)\end{array}$ & $\begin{array}{l}318 \\
(13.1)\end{array}$ & $\begin{array}{l}412 \\
(16.8)\end{array}$ & $508(20.8)$ & $\begin{array}{l}554 \\
(22.6)\end{array}$ & $\begin{array}{l}< \\
0.001\end{array}$ \\
\hline $\begin{array}{l}\text { Self-reported } \\
\text { hyperlipidemia, n (\%) }\end{array}$ & $\begin{array}{l}1732 \\
(17.7)\end{array}$ & $\begin{array}{l}426 \\
(17.5)\end{array}$ & $\begin{array}{l}465 \\
(18.9)\end{array}$ & $467(19.1)$ & $\begin{array}{l}374 \\
(15.3)\end{array}$ & 0.001 \\
\hline Self-reported CHD, n (\%) & $\begin{array}{l}1546 \\
(15.8)\end{array}$ & $\begin{array}{l}342 \\
(14.0)\end{array}$ & $\begin{array}{l}381 \\
(15.5)\end{array}$ & $396(16.2)$ & $\begin{array}{l}427 \\
(17.4)\end{array}$ & 0.012 \\
\hline \multicolumn{7}{|l|}{ Medication use, n (\%) } \\
\hline Antihypertensive drugs & $\begin{array}{l}7772 \\
(79.4)\end{array}$ & $\begin{array}{l}1901 \\
(78.0)\end{array}$ & $\begin{array}{l}1933 \\
(78.7)\end{array}$ & $\begin{array}{l}1977 \\
(80.9)\end{array}$ & $\begin{array}{l}1961 \\
(80.0)\end{array}$ & 0.067 \\
\hline Glucose-lowering drugs & $\begin{array}{l}1144 \\
(11.7)\end{array}$ & $186(7.6)$ & $\begin{array}{l}258 \\
(10.5)\end{array}$ & $327(13.4)$ & $\begin{array}{l}373 \\
(15.2)\end{array}$ & $\begin{array}{l}< \\
0.001\end{array}$ \\
\hline Lipoprotein-lowering drugs & $156(1.6)$ & $51(2.1)$ & $52(2.1)$ & $24(1.0)$ & $29(1.2)$ & $\dot{0} 001$ \\
\hline
\end{tabular}

Q1: quartile 1 (lowest quartile); Q2: quartile 2; Q3: quartile 3; Q4: quartile 4 (highest quartile).

Abbreviations: BaPWV, brachial-ankle pulse wave velocity; BMI, body mass index; SBP, systolic blood pressure; DBP, diastolic blood pressure; CHD, coronary heart disease.

${ }^{*}$ Data are presented as number (\%) or mean \pm SD.

'BMI was calculated as the body weight in kilograms divided by the square of the height in meters $\left(\mathrm{kg} / \mathrm{m}^{2}\right)$.

${ }^{\dagger}$ Hypertension was defined by $\geq 1$ of the following criteria: systolic blood pressure $\geq 140 \mathrm{~mm} \mathrm{Hg}$ or diastolic blood pressure $\geq 90$ or self-reported history of hypertension or use of medications to treat hypertension. 


\begin{tabular}{|c|c|c|c|c|c|c|}
\hline \multirow[t]{3}{*}{ Characteristics $^{*}$} & \multirow[t]{3}{*}{ Total } & \multicolumn{5}{|c|}{$\mathrm{BaPWV}, \mathrm{m} / \mathrm{s}$} \\
\hline & & Q1 & Q2 & Q3 & Q4 & $P$ \\
\hline & & $(<16.00)$ & $\begin{array}{l}(16.00- \\
18.33)\end{array}$ & $\begin{array}{l}(18.33- \\
21.31)\end{array}$ & $(\geq$ & \\
\hline Antiplatelet drugs & $362(3.7)$ & $82(3.4)$ & 84 (3.4) & $86(3.5)$ & $110(4.5)$ & 0.122 \\
\hline \multicolumn{7}{|c|}{ Q1: quartile 1 (lowest quartile); Q2: quartile 2; Q3: quartile 3; Q4: quartile 4 (highest quartile). } \\
\hline \multicolumn{7}{|c|}{$\begin{array}{l}\text { Abbreviations: BaPWV, brachial-ankle pulse wave velocity; BMI, body mass index; SBP, systolic blood } \\
\text { pressure; DBP, diastolic blood pressure; CHD, coronary heart disease. }\end{array}$} \\
\hline \multicolumn{7}{|c|}{${ }^{*}$ Data are presented as number $(\%)$ or mean $\pm \mathrm{SD}$. } \\
\hline \multicolumn{7}{|c|}{$\begin{array}{l}\text { 'BMI was calculated as the body weight in kilograms divided by the square of the height in meters } \\
\left(\mathrm{kg} / \mathrm{m}^{2}\right) \text {. }\end{array}$} \\
\hline \multicolumn{7}{|c|}{$\begin{array}{l}{ }^{+} \text {Hypertension was defined by } \geq 1 \text { of the following criteria: systolic blood pressure } \geq 140 \mathrm{~mm} \mathrm{Hg} \text { or } \\
\text { diastolic blood pressure } \geq 90 \text { or self-reported history of hypertension or use of medications to treat } \\
\text { hypertension. }\end{array}$} \\
\hline
\end{tabular}

\section{Survival analysis according to baPWV status}

During a median follow-up of 20.8 months, there were 138 total first strokes including 123 first ischemic strokes and 15 first hemorrhagic strokes. The Kaplan-Meier curves of the cumulative event rate of first stroke and first ischemic strokes in the baPWV quartiles are shown in Fig. 2. Unadjusted first stroke curves of Q1, Q2 and Q3 were virtually superimposable (Fig. 2A). Subjects in baPWV Q4 displayed significantly greater first stroke than those in Q1, Q2 and Q3 ( $P=0.011$ by log-rank test). Similarly, unadjusted first ischemic stroke curves of Q1, Q2 and Q3 were virtually superimposable (Fig. 2B). Subjects in baPWV Q4 displayed significantly greater first ischemic stroke than those in Q1, Q2 and Q3 ( $P$ $=0.014$ by log-rank test).

\section{Association between baPWV and risk of first stroke (total and subtypes)}

We also tried to use GAM model and penalized spline method to find the nonlinear relationship between baPWV and short-term risk of first stroke (Fig. 3). Adjusted smooth curve showed that the relationship between baPWV and short-term risk of first stroke and first ischemic stroke was curvilinear and reversed L-shaped. On the left of inflection point, the estimated dose-response curve was consistent with a horizontal line. The risk of first stroke and first ischemic stroke increased with the increase of baPWV on the right of inflection point. 
In Table 2, Cox regression analysis was used to estimate the effect of baPWV on short-term risk of first stroke and its subtypes. When baseline baPWV values were assessed as quartiles, in crude model (model 1), the HRs $(95 \% \mathrm{Cl})$ for participants in the Q2 $(16.00$ to $<18.33 \mathrm{~m} / \mathrm{s})$, Q3 $(18.33$ to $<21.31 \mathrm{~m} / \mathrm{s})$ and Q4 $(\geq 21.31 \mathrm{~m} / \mathrm{s}$ ) were 1.18 (95\% Cl: $0.71-1.97), 1.03$ (95\% Cl: $0.61-1.75)$ and 1.89 (95\% Cl: $1.19-3.02)$, respectively compared with those in Q1 (Table 2). Compared with baPWV in Q1-Q3 (<21.31 m/s), baPWV in Q4 ( $\geq 21.31 \mathrm{~m} / \mathrm{s})$ was associated with a $77 \%$ higher risk of first stroke $(\mathrm{OR}=1.77 ; 95 \% \mathrm{Cl}, 1.25-2.50)$. In the multivariate model with adjustment for age, sex, BMI, education status, smoking status, drinking status, labor intensity, sleep quality, hypertension, self-reported of diabetes, self-reported hyperlipidemia, self-reported CHD, antihypertensive drugs, glucose-lowering drugs, lipoprotein-lowering drugs and antiplatelet drugs (model 3), subjects with baPWV $\geq 21.31 \mathrm{~m} / \mathrm{s}$ had a $52 \%$ increased short-term risk of first stroke (HR $=1.52 ; 95 \% \mathrm{Cl}: 1.05-2.21)$ compared with those with baPWV $<21.31 \mathrm{~m} / \mathrm{s}$. 
Table 2

Associations of baseline baPWV with first stroke and its subtypes

\begin{tabular}{|c|c|c|c|c|c|c|c|}
\hline \multirow[t]{2}{*}{ BaPWV, m/s } & \multirow{2}{*}{$\begin{array}{l}\text { Event } \\
\text { (\%) }\end{array}$} & \multicolumn{2}{|l|}{ Model $1^{*}$} & \multicolumn{2}{|l|}{ Model $2^{\dagger}$} & \multicolumn{2}{|l|}{ Model $3^{\ddagger}$} \\
\hline & & $\mathrm{HR}(95 \% \mathrm{Cl})$ & $\begin{array}{l}P \\
\text { value }\end{array}$ & $\mathrm{HR}(95 \% \mathrm{Cl})$ & $\begin{array}{l}P \\
\text { value }\end{array}$ & $\mathrm{HR}(95 \% \mathrm{Cl})$ & $\begin{array}{l}P \\
\text { value }\end{array}$ \\
\hline \multicolumn{8}{|l|}{ First stroke } \\
\hline \multicolumn{8}{|l|}{ Quartiles } \\
\hline Q1 $(<16.00)$ & $\begin{array}{l}27 \\
(1.11)\end{array}$ & Ref. & & Ref. & & Ref. & \\
\hline $\begin{array}{l}\text { Q2 (16.00- } \\
18.33)\end{array}$ & $\begin{array}{l}32 \\
(1.30)\end{array}$ & $\begin{array}{l}1.18(0.71, \\
1.97)\end{array}$ & 0.531 & $\begin{array}{l}1.08(0.65, \\
1.82)\end{array}$ & 0.760 & $\begin{array}{l}1.05(0.63, \\
1.77)\end{array}$ & 0.850 \\
\hline $\begin{array}{l}\text { Q3 (18.33- } \\
21.31)\end{array}$ & $\begin{array}{l}28 \\
(1.15)\end{array}$ & $\begin{array}{l}1.03(0.61 \\
1.75)\end{array}$ & 0.901 & $\begin{array}{l}0.89(0.52, \\
1.53)\end{array}$ & 0.679 & $\begin{array}{l}0.87(0.50 \\
1.50)\end{array}$ & 0.605 \\
\hline Q4 ( $\geq 21.31)$ & $\begin{array}{l}51 \\
(2.08)\end{array}$ & $\begin{array}{l}1.89 \\
3.02)\end{array}$ & 0.007 & $\begin{array}{l}1.48(0.89 \\
2.46)\end{array}$ & 0.132 & $\begin{array}{l}1.46(0.87 \\
2.45)\end{array}$ & 0.147 \\
\hline \multicolumn{8}{|l|}{ Categories } \\
\hline $\begin{array}{l}\text { Q1-Q3 (< } \\
21.31)\end{array}$ & $\begin{array}{l}87 \\
(1.19)\end{array}$ & Ref. & & Ref. & & Ref. & \\
\hline Q4 ( $\geq 21.31)$ & $\begin{array}{l}51 \\
(2.08)\end{array}$ & $\begin{array}{l}1.77(1.25 \\
2.50)\end{array}$ & 0.001 & $\begin{array}{l}1.50(1.04 \\
2.18)\end{array}$ & 0.031 & $\begin{array}{l}1.52(1.05 \\
2.21)\end{array}$ & 0.027 \\
\hline \multicolumn{8}{|c|}{ First ischemic stroke } \\
\hline \multicolumn{8}{|l|}{ Quartiles } \\
\hline Q1 (< 16.00) & $\begin{array}{l}23 \\
(0.94)\end{array}$ & Ref. & & Ref. & & Ref. & \\
\hline $\begin{array}{l}\text { Q2 (16.00- } \\
18.33)\end{array}$ & $\begin{array}{l}28 \\
(1.14)\end{array}$ & $\begin{array}{l}1.21(0.70 \\
2.10)\end{array}$ & 0.499 & $\begin{array}{l}1.11(0.63, \\
1.93)\end{array}$ & 0.725 & $\begin{array}{l}1.06(0.61 \\
1.86)\end{array}$ & 0.826 \\
\hline $\begin{array}{l}\text { Q3 (18.33- } \\
21.31)\end{array}$ & $\begin{array}{l}26 \\
(1.06)\end{array}$ & $\begin{array}{l}1.13(0.64, \\
1.97)\end{array}$ & 0.677 & $\begin{array}{l}0.96(0.54 \\
1.71)\end{array}$ & 0.890 & $\begin{array}{l}0.93(0.52, \\
1.66)\end{array}$ & 0.810 \\
\hline Q4 ( $\geq 21.31)$ & $\begin{array}{l}46 \\
(1.88)\end{array}$ & $\begin{array}{l}2.00(1.21 \\
3.30)\end{array}$ & 0.007 & $\begin{array}{l}1.53(0.89 \\
2.64)\end{array}$ & 0.125 & $\begin{array}{l}1.52(0.87 \\
2.63)\end{array}$ & 0.139 \\
\hline \multicolumn{8}{|l|}{ Categories } \\
\hline \multicolumn{8}{|c|}{ “Model 1: unadjusted. } \\
\hline \multicolumn{8}{|c|}{${ }^{\dagger}$ Model 2: adjusted for age and sex. } \\
\hline $\begin{array}{l}{ }^{\ddagger} \text { Model 3: mo } \\
\text { quality, hyper } \\
\text { antihypertens }\end{array}$ & $\begin{array}{l}+\mathrm{BMI} \\
\text { on, sel } \\
\text { rugs, }\end{array}$ & $\begin{array}{l}\text { cation statu } \\
\text { orted of dia } \\
\text { se-lowering }\end{array}$ & $\begin{array}{l}\text { mokin } \\
\text { es, self } \\
\text { gs, lipc }\end{array}$ & $\begin{array}{l}\text { tatus, drinking } \\
\text { oorted hyperli } \\
\text { otein-lowering }\end{array}$ & $\begin{array}{l}\text { atus, la } \\
\text { emia, s } \\
\text { rugs an }\end{array}$ & $\begin{array}{l}\text { or intensity, sl } \\
\text { f-reported CH } \\
\text { antiplatelet d }\end{array}$ & \\
\hline
\end{tabular}




\begin{tabular}{|c|c|c|c|c|c|c|c|}
\hline \multirow[t]{2}{*}{ BaPWV, m/s } & \multirow{2}{*}{$\begin{array}{l}\text { Event } \\
(\%)\end{array}$} & \multicolumn{2}{|l|}{ Model $1^{*}$} & \multicolumn{2}{|l|}{ Model $2^{\dagger}$} & \multicolumn{2}{|l|}{ Model $3^{\ddagger}$} \\
\hline & & $\mathrm{HR}(95 \% \mathrm{Cl})$ & $\begin{array}{l}P \\
\text { value }\end{array}$ & $\mathrm{HR}(95 \% \mathrm{Cl})$ & $\begin{array}{l}P \\
\text { value }\end{array}$ & $\mathrm{HR}(95 \% \mathrm{Cl})$ & $\begin{array}{l}P \\
\text { value }\end{array}$ \\
\hline $\begin{array}{l}\text { Q1-Q3 (< } \\
21.31)\end{array}$ & $\begin{array}{l}77 \\
(1.05)\end{array}$ & Ref. & & Ref. & & Ref. & \\
\hline Q4 ( $\geq 21.31)$ & $\begin{array}{l}46 \\
(1.88)\end{array}$ & $\begin{array}{l}1.80(1.25 \\
2.59)\end{array}$ & 0.002 & $\begin{array}{l}1.50(1.02 \\
2.22)\end{array}$ & 0.041 & $\begin{array}{l}1.53(1.03 \\
2.26)\end{array}$ & 0.035 \\
\hline \multicolumn{8}{|c|}{ First hemorrhagic stroke } \\
\hline \multicolumn{8}{|l|}{ Quartiles } \\
\hline Q1 (< 16.00) & $\begin{array}{l}4 \\
(0.16)\end{array}$ & Ref. & & Ref. & & Ref. & \\
\hline $\begin{array}{l}\text { Q2 (16.00- } \\
18.33)\end{array}$ & $\begin{array}{l}4 \\
(0.16)\end{array}$ & $\begin{array}{l}1.00(0.25 \\
3.98)\end{array}$ & 0.994 & $\begin{array}{l}0.97(0.24 \\
3.94)\end{array}$ & 0.965 & $\begin{array}{l}1.00(0.24, \\
4.12)\end{array}$ & 0.998 \\
\hline $\begin{array}{l}\text { Q3 (18.33- } \\
21.31)\end{array}$ & $\begin{array}{l}2 \\
(0.08)\end{array}$ & $\begin{array}{l}0.50(0.09 \\
2.74)\end{array}$ & 0.425 & $\begin{array}{l}0.48(0.08 \\
2.74)\end{array}$ & 0.408 & $\begin{array}{l}0.49(0.08, \\
2.84)\end{array}$ & 0.426 \\
\hline Q4 ( $\geq 21.31)$ & $\begin{array}{l}5 \\
(0.20)\end{array}$ & $\begin{array}{l}1.27(0.34 \\
4.73)\end{array}$ & 0.721 & $\begin{array}{l}1.18(0.27 \\
5.13)\end{array}$ & 0.824 & $\begin{array}{l}1.26(0.28 \\
5.72)\end{array}$ & 0.764 \\
\hline \multicolumn{8}{|l|}{ Categories } \\
\hline $\begin{array}{l}\text { Q1-Q3 (< } \\
21.31)\end{array}$ & $\begin{array}{l}10 \\
(0.14)\end{array}$ & Ref. & & Ref. & & Ref. & \\
\hline $\mathrm{Q} 4(\geq 21.31)$ & $\begin{array}{l}5 \\
(0.20)\end{array}$ & $\begin{array}{l}1.53(0.52 \\
4.47)\end{array}$ & 0.440 & $\begin{array}{l}1.52(0.48 \\
4.85)\end{array}$ & 0.475 & $\begin{array}{l}1.61(0.50 \\
5.21)\end{array}$ & 0.430 \\
\hline \multicolumn{8}{|c|}{ “Model 1: unadjusted. } \\
\hline \multicolumn{8}{|c|}{${ }^{\dagger}$ Model 2: adjusted for age and sex. } \\
\hline $\begin{array}{l}\text { ₹Model 3: mo } \\
\text { quality, hypert } \\
\text { antihypertensi }\end{array}$ & $\begin{array}{l}\text { + BMI, } \\
\text { ion, self } \\
\text { drugs, gl }\end{array}$ & $\begin{array}{l}\text { Ication status } \\
\text { oorted of diab } \\
\text { ose-lowering }\end{array}$ & $\begin{array}{l}\text { nokinc } \\
\text { s, self- }\end{array}$ & $\begin{array}{l}\text { tatus, drinkin } \\
\text { oorted hyperli } \\
\text { otein-lowerin }\end{array}$ & $\begin{array}{l}\text { atus, la } \\
\text { emia, s }\end{array}$ & or intensity, s & \\
\hline
\end{tabular}

Similarly, a significantly higher short-term risk of first ischemic stroke $(\mathrm{HR}=1.53 ; 95 \% \mathrm{Cl}$ : $1.03-2.26)$ was found in participants in Q4 ( $\geq 21.31 \mathrm{~m} / \mathrm{s}$ ) compared with participants in Q1-Q3 $(<21.31 \mathrm{~m} / \mathrm{s})$.

Participants in high baPWV ( $\geq 21.31 \mathrm{~m} / \mathrm{s}$ ) had a $61 \%$ higher short-term risk of first hemorrhagic stroke $(\mathrm{HR}=1.61,95 \% \mathrm{Cl}: 0.50-5.21)$, but there did not reach statistical significance.

\section{Sensitivity Analysis}

We generated an E-value to assess the sensitivity to unmeasured confounding. Higher baPWV ( $\geq$ $21.31 \mathrm{~m} / \mathrm{s})$ are associated with an increase in first stroke by uninvite analysis (HR, 1.77, 95\% Cl: 1.25- 
2.50) and multivariable analysis ( $\mathrm{HR}, 1.52,95 \% \mathrm{Cl}: 1.05-2.21)$. The E-value was 2.94 , meaning that residual confounding could explain the observed association if there exists an unmeasured covariate having a relative risk association $\geq 2.94$ with both first stroke and baPWV. Therefore, it is unlikely that an unmeasured or unknown confounder would have a substantially greater effect on first stroke (relative risk exceeding 2.94) than these known risk factors. Consistently, the primary findings were robust, unless an unmeasured confounder existed with a higher relative risk of first ischemic stroke, with an HR higher than 3.00 .

In addition, stratified analyses were performed to assess the association of baseline baPWV ( $\geq$ $21.31 \mathrm{~m} / \mathrm{s}$ vs. Q1-3, $<21.31 \mathrm{~m} / \mathrm{s}$ ) with first stroke and first ischemic stroke in various subgroups (Figure $\mathrm{S} 1$ and Figure S2). Regardless of subgroup, compared with low baPWV $(<21.31 \mathrm{~m} / \mathrm{s})$, high baPWV ( $\geq$ $21.31 \mathrm{~m} / \mathrm{s}$ ) was positively associated with the short-term risk of first stroke and first ischemic stroke. As shown in Fig. 4, compared with low baPWV $(<21.31 \mathrm{~m} / \mathrm{s})$, high baPWV $(\geq 21.31 \mathrm{~m} / \mathrm{s})$ was associated with a 3.30-fold (95\% Cl: 1.13-9.62) increase in the short-term risk of first stroke in the subgroup with $\mathrm{SBP}<140 \mathrm{~mm} \mathrm{Hg}$, whereas the association was weaker in the subgroup with $\mathrm{SBP} \geq 140 \mathrm{~mm} \mathrm{Hg}(\mathrm{HR}=$ $1.29,95 \% \mathrm{Cl}: 0.86-1.93)$. The magnitude of the association between the baPWV and the incidence of stroke tended to be greater in DBP $<90 \mathrm{~mm} \mathrm{Hg}$ group ( $\mathrm{HR}=1.78 ; 95 \% \mathrm{Cl}: 1.06-2.98)$ as compared with that in the subgroup with $\mathrm{DBP} \geq 90 \mathrm{~mm} \mathrm{Hg}(\mathrm{HR}=1.09,95 \% \mathrm{Cl}: 0.62-1.91)$. Similarly, the association of high baPWV with the short-term risk of first ischemic stroke was significant in hypertensive patients with $\mathrm{SBP}<140 \mathrm{~mm} \mathrm{Hg}(\mathrm{HR}=4.14,95 \% \mathrm{Cl}: 1.35-12.68)$ and $\mathrm{DBP}<90 \mathrm{~mm} \mathrm{Hg}(\mathrm{HR}=1.86,95 \% \mathrm{Cl}: 1.07-3.23)$ (Figure S1). There was no association between baPWV and the short-term risk of first ischemic stroke among patients with inadequate hypertension control (Figure S1). These findings suggest that the magnitude of the association between the baPWV and the incidence of first stroke tended to be greater in the subgroup with controlled BP as compared with that in the subgroup with uncontrolled BP.

\section{Discussion}

We found that high baPWV levels ( $\geq 21.31 \mathrm{~m} / \mathrm{s}$ ) were associated with increased the short-term risk of first stroke among Chinese hypertensive adults, compared to low baPWV levels, supporting a reversed Lshaped association between baPWV and risk of first stroke. Sensitivity analyses showed consistent results. These results suggest that a baPWV of $21.31 \mathrm{~m} / \mathrm{s}$ as the threshold for high risk in hypertensive patients. Furthermore, our study extends the results of previously published related studies by demonstrating that the association between baPWV and first stroke risk could be modified by SBP and DBP levels. Our findings warrant additional investigation.

Several previous studies have reported the association between baPWV and CVD and cerebrovascular diseases. A meta-analysis including 14673 Japanese participants without preexisting CVD established baPWV as an independent predictor of the risk of development of $\mathrm{CVD}^{[22]}$. Yong Bum Kim et al ${ }^{[16]}$ used data from 1282 Korea participants and found that baPWV with both acute and chronic cerebral small vessel disease. Ting Li et al ${ }^{[23]}$ indicated that increased baPWV were objective indicators of increased risk of ischemic stroke in patients with type 2 diabetes. These studies showed a linear association between 
baPWV and CVD. Of note, to our knowledge, only one study investigated the baPWV-stroke association in hypertension patients. Yun Song et al ${ }^{[12]}$ conducted a prospective cohort study of 3310 Chinese hypertensive adults and found that high baPWV $(\geq 20.85 \mathrm{~m} / \mathrm{s})$ was significantly associated with an increased risk of first stroke.

Our study provides some new insights into this field. First, we found that high baPWV levels $(\geq$ $21.31 \mathrm{~m} / \mathrm{s}$ ) were associated with increased first stroke risk among Chinese hypertensive patients, supporting a threshold effect of baPWV on stroke. Toshiaki Ohkuma et al[ ${ }^{[24]}$ proposed that the optimal cutoff value of baPWV for CVD in patients with hypertension was $18.3 \mathrm{~m} / \mathrm{s}$. Moreover, the threshold of baPWV varies by population and health conditions ${ }^{[25-28]}$. Our study findings have important clinical implications. To date, none of the stroke prevention guidelines have considered PWV as a predictor in risk assessment. Our findings raised that individuals with baPWV $\geq 20 \mathrm{~m} / \mathrm{s}$ were at high risk of developing stroke among patients with hypertension. Several possible mechanisms for could explain the association between baPWV and incidence of first stroke. BaPWV, is not only considered as a marker of atherosclerosis, but also has a pathophysiologic role, which could promote the development of hypertension, macro/microvascular damage, and brain structural injury ${ }^{[29,30]}$. Moreover, baPWV may be associated with stroke risk factors which in turn are important predictors of stroke ${ }^{[31]}$. Additionally, high baPWV is associated with the development of stroke through endothelial dysfunction, extracellular matrix disorder, elevated endothelial permeability, mechanical force on the inner wall of blood vessels, and vascular aging ${ }^{[19,32]}$. Possibly, arterial stiffness may also increase in patients with endothelial dysfunction, oxidative stress, and inflammatory conditions, which could increase the risk of stroke $\mathrm{e}^{[15,33]}$. However, more studies are needed to confirm our findings and further elucidate the specific biological mechanisms.

Second, the magnitude of the effect of high baPWV on the risk of development of stroke was greater in hypertensive patients with well controlled BP. There was no association between baPWV and risk of stroke among patients with inadequate hypertension control. Consistent with our results, Toshiaki Ohkuma et al ${ }^{[22]}$ also found that the association between baPWV and the risk of development of CVD was stronger in the participants without diabetes mellitus or hypertension than in those with either/both of these diseases. However, one previous study reported that participants with high baPWV and inadequate hypertension control had the highest risk of stroke compared with other groups. Differences in ethnics and methodological variations in baPWV measurements may possibly explain this discrepancy. Several considerations may explain the presence of this hypertension control paradox. While inadequate hypertension control is a major risk factor for stroke, subjects with the risk factor are also supposed to have higher baPWV levels in both the case group and the control group. Consequently, residual confoundings were inevitable when we analyzed association of high baPWV and risk of stroke in subjects with the factor. One hypothesis that has been proposed to explain this paradox is that by virtue of inadequate hypertension control, patients are offered earlier and more aggressive treatments to manage stroke risk factors. This may be related to the earlier, more intensive and closely monitored use of antiplatelet and anti-dyslipidaemic medications. Therefore, the findings may propose the applicability 
of baPWV measurement for prediction of stroke development, especially in subjects with a low atherosclerotic disease risk.

Potential limitations of our study should also be noted. First, the potential for residual confounding exists, as with all observational studies. Specifically, blood glucose and lipids could not be measured. We used the E-value sensitivity analysis to quantify the potential implications of unmeasured confounders and found that an unmeasured confounder was unlikely to explain the entirety of baPWV effect. Second, our study was underpowered to investigate the association between baPWV and risk of first hemorrhagic stroke. Third, the participants were all hypertensive from China; thus, the generalizability to general population or other ethics should be further verified. Fourth, the measurements of baPWV were only conducted at baseline. More frequent measurements of baPWV would provide more useful information. Fifth, follow-up time in the present study was short. However, the significant association between baPWV and risk of stroke was still observed, indicating high baPWV could increase the short-term risk of stroke. Despite these limitations, the strengths of the present study were the inclusion of a large number of participants, which allowed for sufficient statistical power to detect differences, the adjustments for confounders, and the subgroup analyses. Moreover, the threshold effect of baPWV on first stroke risk was observed. Furthermore, our results suggest that measurement of the baPWV may provide additional predictive information for future development of stroke in hypertensive patients with controlled BP.

\section{Conclusion}

In summay, high baPWV levels ( $\geq 21.31 \mathrm{~m} / \mathrm{s}$ ) were associated with increased the short-term risk of first stroke among Chinese hypertensive adults, compared to low baPWV levels, supporting a reversed Lshaped association between baPWV and risk of first stroke. Our findings may further propose the applicability of baPWV measurement for prediction of stroke development, especially in subjects with a low atherosclerotic disease risk.

\section{Abbreviations}

CVD, cardiovascular; cfPWV, carotid-femoral pulse wave velocity; BP: blood pressure; CHRS, China Hypertension Registry Study; $A B I$, ankle brachial index; CHD, coronary heart disease; ANOVA, analysis of Variance; BaPWV, brachial-ankle pulse wave velocity; BMI, body mass index; SBP, systolic blood pressure; DBP, diastolic blood pressure; $\mathrm{CHD}$, coronary heart disease; $\mathrm{Cl}$, confidence interval; $\mathrm{HR}$, hazard ratio.

\section{Declarations}

\section{Ethics approval and consent to participate}

The study was conducted in accordance with the declaration of helsinki and was approved by the Institute of Biomedicine, Anhui Medical University, Hefei, China. Informed written consent was obtained from all patients before their enrollment in this study. 


\section{Consent for publication}

Not applicable.

\section{Availability of data and materials}

The datasets used and/or analyzed in the current study are available from the corresponding author upon reasonable request.

\section{Competing interests}

The authors declare they have no actual or potential competing financial interests.

\section{Funding}

The study was supported by funding from the following: the National Key Research and Development Program of China [2016YFE0205400, 2018ZX09739010, 2018ZX09301034003]; the Science and Technology Program of Guangdong, China [2020B121202010]; the Science and Technology Planning Project of Guangzhou, China [201707020010]; the Science, Technology and Innovation Committee of Shenzhen, China [GJHS20170314114526143, JSGG20180703155802047]; the Economic, Trade and Information Commission of Shenzhen Municipality, China [20170505161556110, 20170505160926390, 201705051617070]; the National Natural Science Foundation of China [81960074, 81500233]; Jiangxi Outstanding Person Foundation [20192BCBL23024] and the major projects of the Science and Technology Department, Jiangxi [20171BAB205008].

\section{Authors' contributions}

Conceptualization and methodology: XPX, YH, JPL. Software: LHH, LSL. Validation: YS, YZ. Formal analysis: LHH, YZ. Investigation: LHH, CLB, LSL, BYW, CQF, HM, XH, YZ. Data curation: LSL, YS, YZ. Writing (original draft preparation): LHH. Writing (review and editing):CLB, LSL, YS, BYW, CQF, HM, XH, YZ, XPX, XBW, YH, JPL. Supervision: XPX, YH, JPL. Project administration: CLB, LSL, YS, BYW, HM. Funding acquisition: $\mathrm{XPX}, \mathrm{XH}$. All authors read and approved the final manuscript.

\section{Acknowledgements}

We acknowledge the contribution the all staff who participated in this study as well as the study participants who shared their time with us.

\section{References}

1. Wang W, Jiang B, Sun H, et al. Prevalence, Incidence, and Mortality of Stroke in China: Results from a Nationwide Population-Based Survey of 480687 Adults[J]. Circulation. 2017, 135(8): 759-771.

2. Global, regional, and national burden of stroke, 1990-2016: a systematic analysis for the Global Burden of Disease Study 2016[J]. Lancet Neurol. 2019, 18(5): 439-458. 
3. Meschia J F, Bushnell C, Boden-Albala B, et al. Guidelines for the primary prevention of stroke: a statement for healthcare professionals from the American Heart Association/American Stroke Association[J]. Stroke. 2014, 45(12): 3754-3832.

4. Huo Y, Li J, Qin X, et al. Efficacy of folic acid therapy in primary prevention of stroke among adults with hypertension in China: the CSPPT randomized clinical trial[J]. JAMA. 2015, 313(13): 1325-1335.

5. Hoshino T, Sissani L, Labreuche J, et al. Prevalence of Systemic Atherosclerosis Burdens and Overlapping Stroke Etiologies and Their Associations With Long-term Vascular Prognosis in Stroke With Intracranial Atherosclerotic Disease[J]. JAMA Neurol. 2018, 75(2): 203-211.

6. Cavalcante J L, Lima J A, Redheuil A, et al. Aortic stiffness: current understanding and future directions[J]. J Am Coll Cardiol. 2011, 57(14): 1511-1522.

7. Vlachopoulos C, Aznaouridis K, Terentes-Printzios D, et al. Prediction of cardiovascular events and all-cause mortality with brachial-ankle elasticity index: a systematic review and meta-analysis[J]. Hypertension. 2012, 60(2): 556-562.

8. Tomiyama H, Matsumoto C, Shiina K, et al. Brachial-Ankle PWV: Current Status and Future Directions as a Useful Marker in the Management of Cardiovascular Disease and/or Cardiovascular Risk Factors[J]. J Atheroscler Thromb. 2016, 23(2): 128-146.

9. Tanaka H, Munakata M, Kawano Y, et al. Comparison between carotid-femoral and brachial-ankle pulse wave velocity as measures of arterial stiffness[J]. J Hypertens. 2009, 27(10): 2022-2027.

10. Mancia G, Fagard R, Narkiewicz K, et al. 2013 ESH/ESC Guidelines for the management of arterial hypertension: the Task Force for the management of arterial hypertension of the European Society of Hypertension (ESH) and of the European Society of Cardiology (ESC)[J]. J Hypertens. 2013, 31(7): 1281-1357.

11. Hu L, Zhang Y, Huang X, et al. Associations between Blood Pressure Indices and Brachial-ankle Pulse Wave Velocity in Treated Hypertensive Adults: results from the China Stroke Primary Prevention Trial (CSPPT)[J]. Sci Rep. 2019, 9(1): 8178.

12. Song $Y, X u B, X u R$, et al. Independent and Joint Effect of Brachial-Ankle Pulse Wave Velocity and Blood Pressure Control on Incident Stroke in Hypertensive Adults[J]. Hypertension. 2016, 68(1): 4653.

13. Vlachopoulos C, Aznaouridis K, Stefanadis C. Prediction of cardiovascular events and all-cause mortality with arterial stiffness: a systematic review and meta-analysis[J]. J Am Coll Cardiol. 2010, 55(13): 1318-1327.

14. Ninomiya T, Kojima I, Doi Y, et al. Brachial-ankle pulse wave velocity predicts the development of cardiovascular disease in a general Japanese population: the Hisayama Study[J]. J Hypertens. 2013, 31(3): 477-483, 483.

15. Kim J, Song T J, Kim E H, et al. Brachial-ankle pulse wave velocity for predicting functional outcome in acute stroke[J]. Stroke. 2014, 45(8): 2305-2310.

16. Kim Y B, Park K, Chung P, et al. Brachial-ankle pulse wave velocity is associated with both acute and chronic cerebral small vessel disease[J]. Atherosclerosis. 2016, 245: 54-59. 
17. Chen S C, Chang J M, Liu W C, et al. Brachial-ankle pulse wave velocity and rate of renal function decline and mortality in chronic kidney disease[J]. Clin J Am Soc Nephrol. 2011, 6(4): 724-732.

18. Yamashina A, Tomiyama $\mathrm{H}$, Takeda $\mathrm{K}$, et al. Validity, reproducibility, and clinical significance of noninvasive brachial-ankle pulse wave velocity measurement[J]. Hypertens Res. 2002, 25(3): 359364.

19. Liu Z, Yang Y, Zhang Y, et al. Association of brachial-ankle pulse wave velocity and carotid plaque in Chinese hypertensive adults: effect modification by age[J]. Hypertension Research. 2020, 43(8): 808816.

20. Sun P, Liu L, Liu C, et al. Carotid Intima-Media Thickness and the Risk of First Stroke in Patients With Hypertension[J]. Stroke. 2020, 51(2): 379-386.

21. Vanderweele T J, Ding P. Sensitivity Analysis in Observational Research: Introducing the E-Value[J]. Ann Intern Med. 2017, 167(4): 268-274.

22. Ohkuma T, Ninomiya $T$, Tomiyama $H$, et al. Brachial-Ankle Pulse Wave Velocity and the Risk Prediction of Cardiovascular Disease[J]. Hypertension. 2017, 69(6): 1045-1052.

23. Li T, Wu X J, Chen X M, et al. Ankle-brachial index and brachial-ankle pulse wave velocity are risk factors for ischemic stroke in patients with Type 2 diabetes[J]. Neural Regen Res. 2017, 12(11): 18531859.

24. Ohkuma T, Tomiyama H, Ninomiya T, et al. Proposed Cutoff Value of Brachial-Ankle Pulse Wave Velocity for the Management of Hypertension[J]. Circ J. 2017, 81(10): 1540-1542.

25. Morimoto S, Yurugi T, Aota Y, et al. Prognostic significance of ankle-brachial index, brachial-ankle pulse wave velocity, flow-mediated dilation, and nitroglycerin-mediated dilation in end-stage renal disease[J]. Am J Nephrol. 2009, 30(1): 55-63.

26. Nakamura M, Yamashita T, Yajima J, et al. Brachial-ankle pulse wave velocity as a risk stratification index for the short-term prognosis of type 2 diabetic patients with coronary artery disease[J]. Hypertens Res. 2010, 33(10): 1018-1024.

27. Meguro T, Nagatomo Y, Nagae A, et al. Elevated arterial stiffness evaluated by brachial-ankle pulse wave velocity is deleterious for the prognosis of patients with heart failure[J]. Circ J. 2009, 73(4): 673-680.

28. Kitahara T, Ono K, Tsuchida A, et al. Impact of brachial-ankle pulse wave velocity and ankle-brachial blood pressure index on mortality in hemodialysis patients[J]. Am J Kidney Dis. 2005, 46(4): 688696.

29. Zeki A H A, Newman A B, Simonsick E, et al. Pulse wave velocity and cognitive decline in elders: the Health, Aging, and Body Composition study[J]. Stroke. 2013, 44(2): 388-393.

30. Webb A J, Simoni M, Mazzucco S, et al. Increased cerebral arterial pulsatility in patients with leukoaraiosis: arterial stiffness enhances transmission of aortic pulsatility[J]. Stroke. 2012, 43(10): 2631-2636.

31. Liu X, Sun N, Yu T, et al. The Independent and Joint Association of Blood Pressure, Serum Total Homocysteine, and Fasting Serum Glucose Levels With Brachial-Ankle Pulse Wave Velocity in 
Chinese Hypertensive Adults[J]. Int Heart J. 2016, 57(5): 627-633.

32. A C P, B M K. Arterial stiffness, atherosclerosis and cardiovascular risk: Pathophysiologic mechanisms and emerging clinical indications.[J]. Vascular Pharmacology. 2016, 77: 1-7.

33. Saji N, Kimura K, Kawarai T, et al. Arterial stiffness and progressive neurological deficit in patients with acute deep subcortical infarction[J]. Stroke. 2012, 43(11): 3088-3090

\section{Figures}


China Hypertension Registry

Study in Rongcheng

$$
(\mathrm{n}=111297)
$$

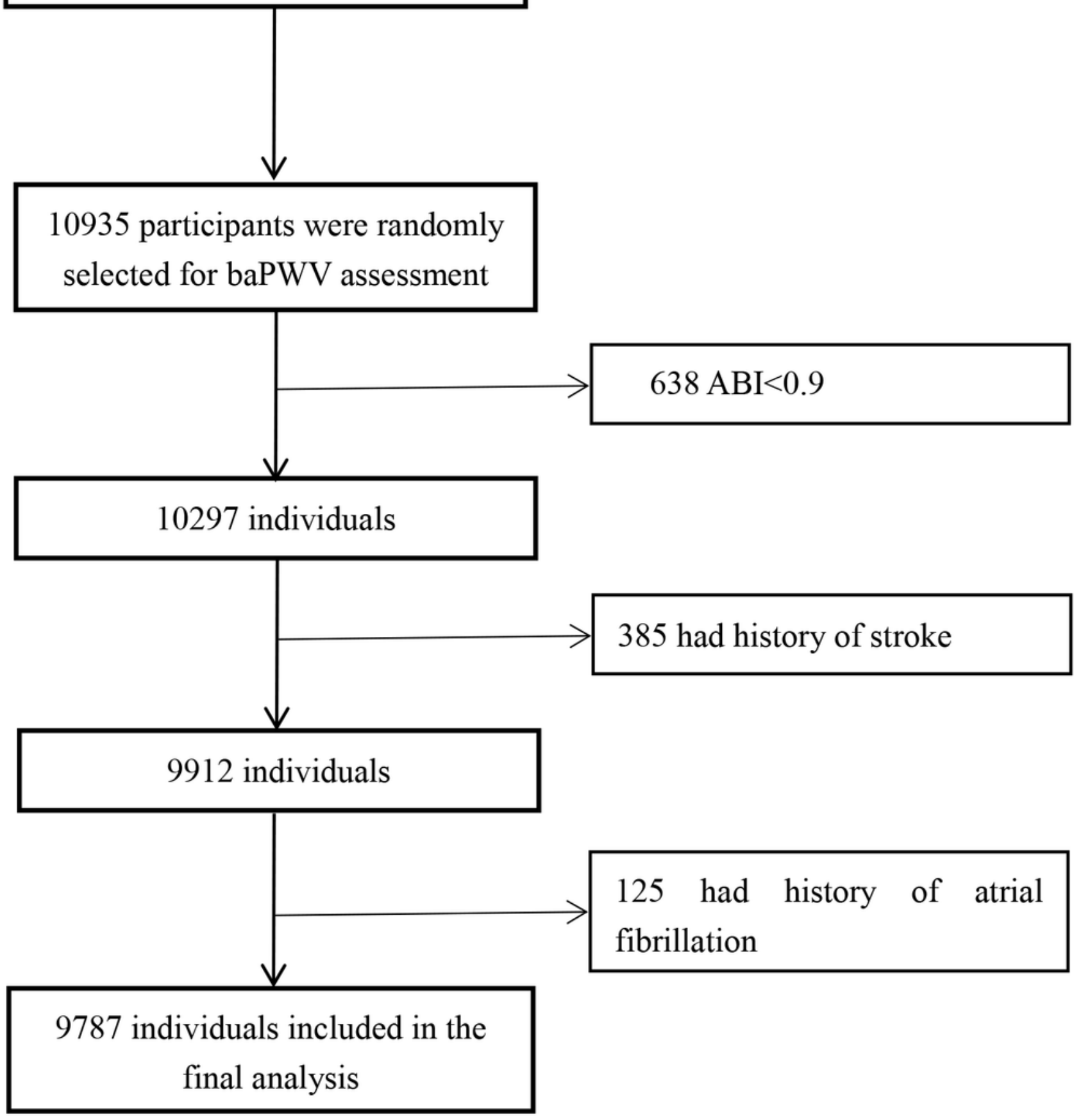

Figure 1

Flow chart of study populations. 


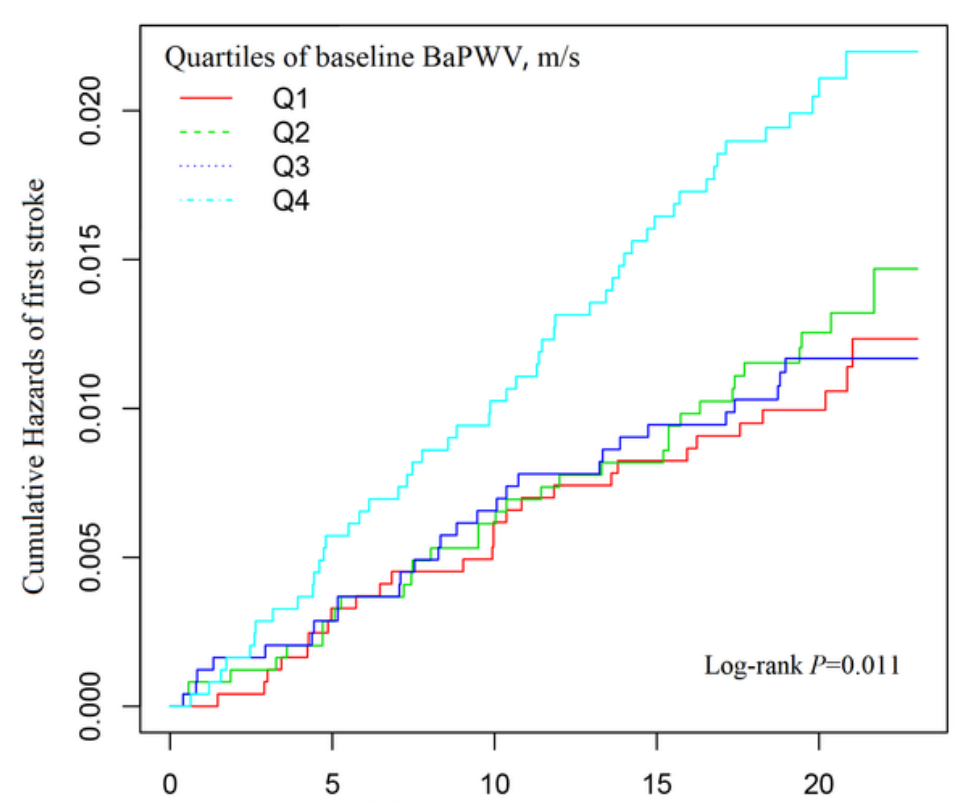

(A) Follow-up (months)

No. at risk by baPWV quartiles $(\mathrm{m} / \mathrm{s})$

$\begin{array}{llllll}\text { Q1 }(<16.00) & 2436 & 2428 & 2421 & 2412 & 1671 \\ \text { Q2 (16.00-18.33) } & 2455 & 2448 & 2440 & 2431 & 1689 \\ \text { Q3 }(18.33-21.31) & 2445 & 2438 & 2429 & 2415 & 1701 \\ \text { Q4 }(\geq 21.31) & 2451 & 2437 & 2426 & 2409 & 1644\end{array}$

Figure 2

Kaplan-Meier survival curves for unadjusted first stroke (A) and first ischemic stroke (B) according to quartiles of baseline brachial-ankle pulse wave velocity (BaPWV).
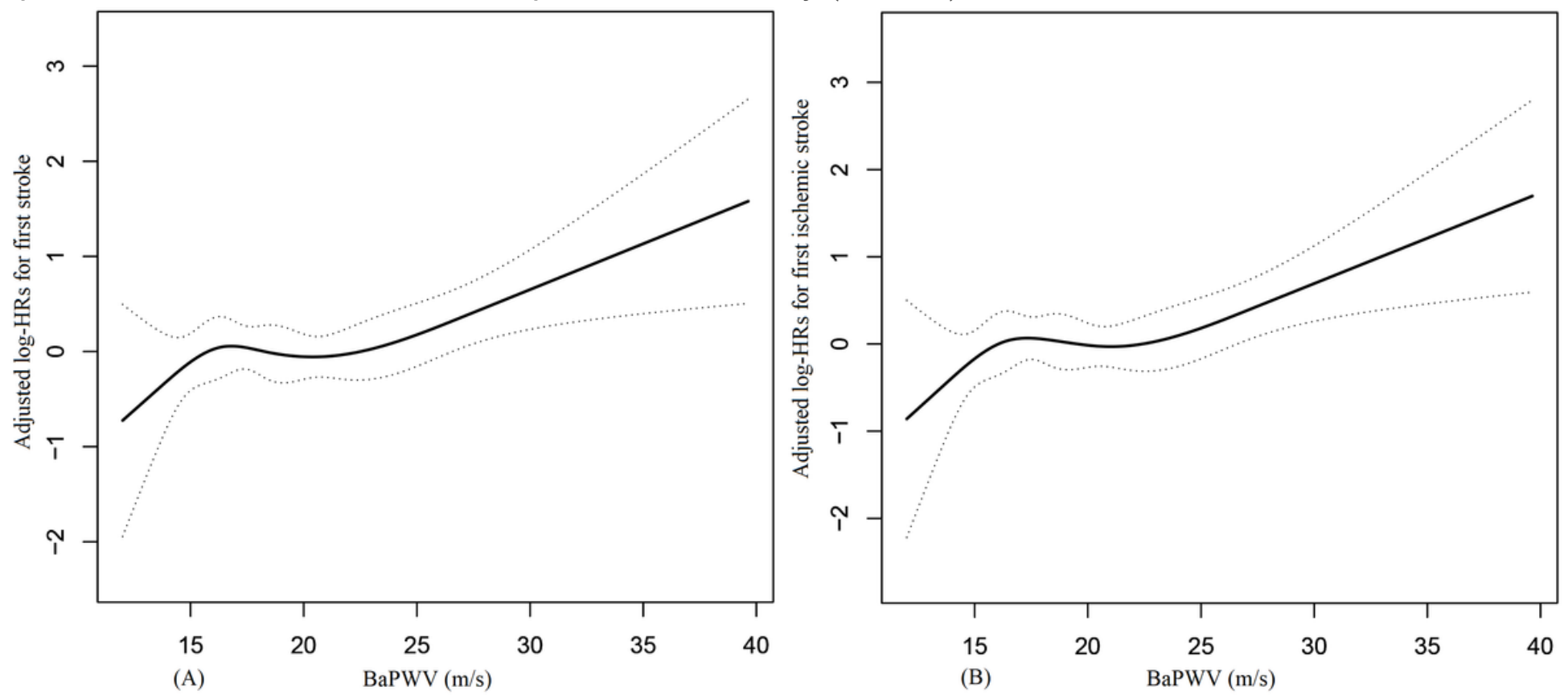

Figure 3 
The association between baseline baPWV and incident risk of first stroke (A) and first ischemic stroke (B). Adjusted for age, sex, BMI, education status, smoking status, drinking status, labor intensity, sleep quality, hypertension, self-reported of diabetes, self-reported hyperlipidemia, self-reported CHD, antihypertensive drugs, glucose-lowering drugs, lipoprotein-lowering drugs and antiplatelet drugs.

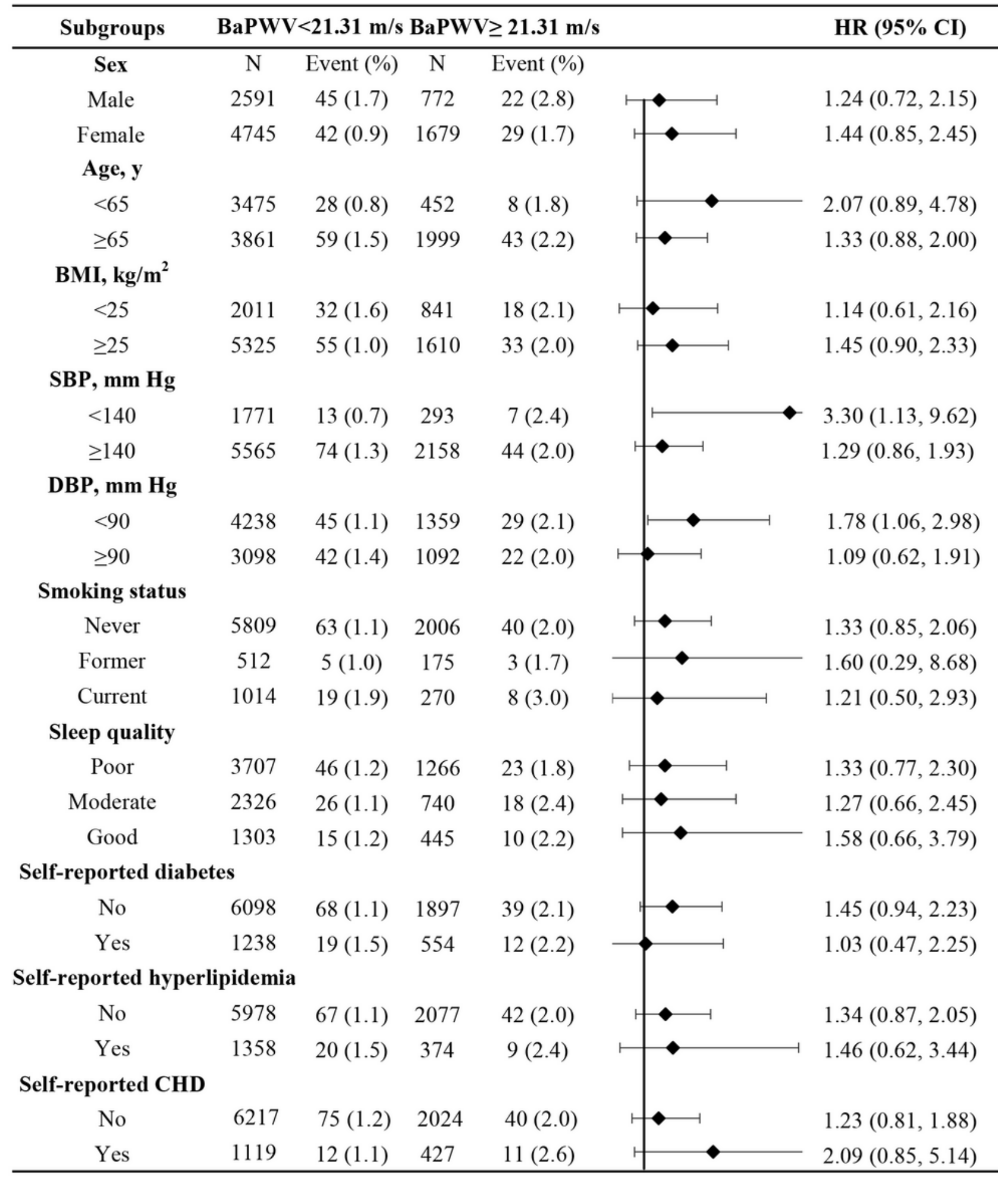

Figure 4 
The association between baseline baPWV (Q4 vs. Q1-Q3) and incident risk of first stroke in various subgroups. Adjusted for age, sex, BMI, SBP, DBP, education status, smoking status, drinking status, labor intensity, sleep quality, self-reported of diabetes, self-reported hyperlipidemia, self-reported CHD, antihypertensive drugs, glucose-lowering drugs, lipoprotein-lowering drugs and antiplatelet drugs, except for the stratifying variable.

\section{Supplementary Files}

This is a list of supplementary files associated with this preprint. Click to download.

- FigureS1.png

- Supplementalfigurelegends.docx 